\title{
Tipificación de Geranium murcicum Cav. (Geraniaceae)
}

\author{
Pedro Pablo Ferrer-Gallego
}

Servicio de Espacios Naturales y Biodiversidad. Centro para la Investigación y la Experimentación Forestal. Generalitat Valenciana (CIEF). Avda. Comarques del País Valencià, 114, E-46930, Quart de Poblet, Valencia.

\section{Resumen}

Correspondencia

P.P. Ferrer-Gallego

E-mail: flora.cief@gva.es

Recibido: 22 marzo 2012

Aceptado: 26 mayo 2012

Publicado on-line: 25 junio 2012

Se designa un lectótipo (iconolectótipo) para Geranium murcicum Cav. (Geraniaceae).

Palabras clave: Nomenclatura, lectótipo, Geranium murcicum, Geraniaceae.

\author{
Abstract \\ Tipification of Geranium murcicum Cav. (Geraniaceae) \\ A lectotype (iconolectotype) is designated for Geranium murcicum \\ Cav. (Geraniaceae). \\ Key words: Nomenclature, lectotype, Geranium murcicum, \\ Geraniaceae.
}

\section{Introducción}

El género Erodium L'Hér. constituye un grupo vegetal integrado aproximadamente por unas 60 especies, mostrando su mayor diversidad en la cuenca Mediterránea (Knuth, 1912; Guittonneau, 1972). En la actualidad, la especie descrita por Cavanilles como Geranium murcicum (Geraniaceae) a finales del siglo XVIII, se reconoce dentro de la variabilidad infraespecífica de Erodium chium (L.) Willd., interpretada por algunos autores a nivel varietal (Guittonneau, 1972; Bolòs \& Vigo, 1990) y por otros (Webb \& Chater, 1968) con rango subespecífico bajo el nombre prioritario $E$. chium subsp. littoreum (Léman) Ball. El propio Cavanilles (1788) indica que se trata de una especie próxima a G. chium L., y así ha sido tradicionalmente reconocida su independencia a lo largo del tiempo, a pesar de no estar del todo clara su separación (Rosselló \& Sáez, 2000) y clasificación dentro de la especie linneana.

Esta planta se distribuye preferentemente por territorios próximos al litoral, desde el SE de España (Devesa, 1987; Sánchez-Gómez \& Guerra, 2003) hasta el S de Francia, también presente en las Islas Baleares (Fraga \& al., 2004) y alcanzando la isla de Córcega (Jeanmonod \& Gamisans, 2007). Habita en comunidades ruderales, campos de cultivo y sus ribazos, barbechos, márgenes de caminos, taludes y terrenos removidos. Se diferencia morfológicamente de la forma típica de $E$. chium por ser una planta vivaz, con hojas más profundamente divididas, tripartidas o trisectas, nectarios verdes y estigmas amarillos, mientras que la var. chium suele comportarse como una planta anual o bienal, menos densamente pubescente, con hojas trilobadas, nectarios marrones y estigmas rosáceos. Al parecer esta relación de caracteres se mantiene en las plantas cultivadas bajo condiciones controladas, aunque trabajos experimentales demuestran que la descendencia fruto del cruce entre estas dos variedades es fértil (Guittonneau, 1972).

Desde el punto de vista taxonómico, la validez de la planta cavanillesiana no está exenta de controversia, en parte debido según nuestra opinión por no haberse abordado hasta el momento la respectiva tipificación, lo que puede haber inducido a una mala interpretación del taxón. 


\section{Tipificación}

Geranium murcicum Cav., Diss. 5: 272, tab. 126 fig. 1 (inter 23 et 26-VII-1788)

$\equiv$ Erodium murcicum (Cav.) Willd., Sp. Pl. 3(1): 636 (1800); Erodium chium var. murcicum (Cav.) Nyman, Consp. Fl. Eur.: 139 (1878); Erodium chium proles murcicum (Cav.) Rouy, Fl. Fr. 4: 120 (1897); Erodium chium var. murcicum (Cav.) Rouy in Willk., Suppl. Prodr. Fl. Hisp.: 266 (1893), comb. superfl.; Erodium littoreum var. murcicum (Cav.) P. Fourn., Quatre Fl. Fr.: 625 (1937), comb. Illeg.

= Erodium littoreum Léman ex DC., Fl. Franc. (DC. \& Lamarck), ed. 3, 4: 843 (1805); Erodium chium subsp. littoreum (Léman) Ball, Jour. Linn. Soc. London (Bot.) 16: 387 (1878); Erodium chium proles littoreum (Léman) Rouy, Fl. Fr. 4: 120 (1897).

Ind. loc.: "Habitat in Hispania prope Murciam".

Lectotypus (hic designatus): [icon]; Cavanilles, Diss. 5, tab. 126, fig. 1 (inter 23 et 26-VII-1788), Geranium murcicum Cav. (Fig. 1).

Respecto al material recolectado de G. murcicum por el propio Cavanilles, al igual que Garilleti (1993) solo hemos localizado un pliego en el Real Jardín Botánico de Madrid (MA 71732), este pliego conserva un ejemplar en fruto y restos de flores, recortes de la lámina de Cavanilles y de la descripción original, lleva también una etiqueta manuscrita a tinta con letra de Cavanilles, donde aparece escrito: "Geranium murcicum // in Saguntini montis radice". Este material fue considerado en una etiqueta de revisión de C. Vicioso, sin fecha, como el "Typus" del nombre cavanillesiano, propuesta que nunca fue publicada y por lo tanto resulta no efectiva [art. 7.10 del CINB (McNeill $\&$ al., 2006)]. Por otro lado, el material que contiene este pliego no puede ser considerado original de Cavanilles, pues la indicación sobre el lugar de recolección que aparece en la etiqueta "Saguntini" pertenece a una localidad de la provincia de Va- lencia, y no es mencionada en el protólogo de la especie.

\section{Referencias}

Bolòs O de \& Vigo J. 1990. Flora dels Països Catalans. Vol. 2. Editorial Barcino. Barcelona.

Cavanilles AJ. 1788. Quinta dissertatio botanica. De Sterculia, Kleinhovia, Ayenia, Buttneria, Bombace, Adansonia, Crinodendro, Aytonia, Malachodendro, Stewartia et Napaea. Accedit praecedentium dissertatiorum Mantissa, 36 Tabulis aere incisis ornata. Paris.

Devesa JA. 1987. Geraniaceae. En: Valdés B, Talavera $S$ \& Fernández-Galianao $\mathrm{E}$ (eds.). Flora Vascular de Andalucía Occidental 2: 271-284. Ketrés ed. Barcelona.

Fraga P, Mascaró C, Carreras D, García Ò, Pallicer X, Pons M, Seoane M \& Truyol M. 2004. Catàleg de la flora vascular de Menorca. Collecció Recerca 9. Ed. IME. Ciutadella de Menorca.

Garilleti R. 1993. Herbarium cavanillesianum seu enumeratio plantarum exsiccatarum aliquo ad novitatis cavanillesianas pertinentium, quae in Horti Regii Matritensis atque Londinensis Societatis Linnaeanae herbariis asservantur. Fontqueria $38: 6-248$.

Guittonneau GG. 1972. Contribution à l'étude biosystématique du genre Erodium L'Hér. dans le bassin méditérranéen occidental. Boissiera 20: 1-154.

Jeanmonod D \& Gamisans J. 2007. Flora corsica. Edisud. Aix-en-Provence.

Knuth R. 1912. Geraniaceae. In: Engler A. (ed.). Das Pflanzenreich. IV.129 (Heft 53). Wilhelm Engelmann, Leipzing: 1-640.

McNeill J, Barrie FR, Burdet HM, Demoulin V, Hawksworth DL, Marhold K, Nicolson DH, Prado J, Silva PC, Skog JE, Wiersema JH \& Turland NJ. (eds.). 2006. International Code of Botanical Nomenclature (Vienna Code) adopted by the Seventeenth International Botanical Congress Vienna, Austria, July 2005. Regnum Vegetabile 146. A.R.G. Gantner Verlag, Ruggell, Liechtenstein.

Rosselló JA \& Sáez LI. 2000. Index Balearicum: An annotated check-list of the vascular plants described from the Balearic Islands. Collect. Bot. (Barcelona) 25(1): 1-192.

Sánchez-Gómez P \& Guerra J. (eds.). 2003. Nueva flora de Murcia. Plantas Vasculares. DM Editorial. Murcia.

Webb DA \& Chater AO. 1968. Erodium L'Hér. In: Tutin TG \& al (eds.). Flora Europaea 2: 199-204. Cambridge University Press, Cambridge. 


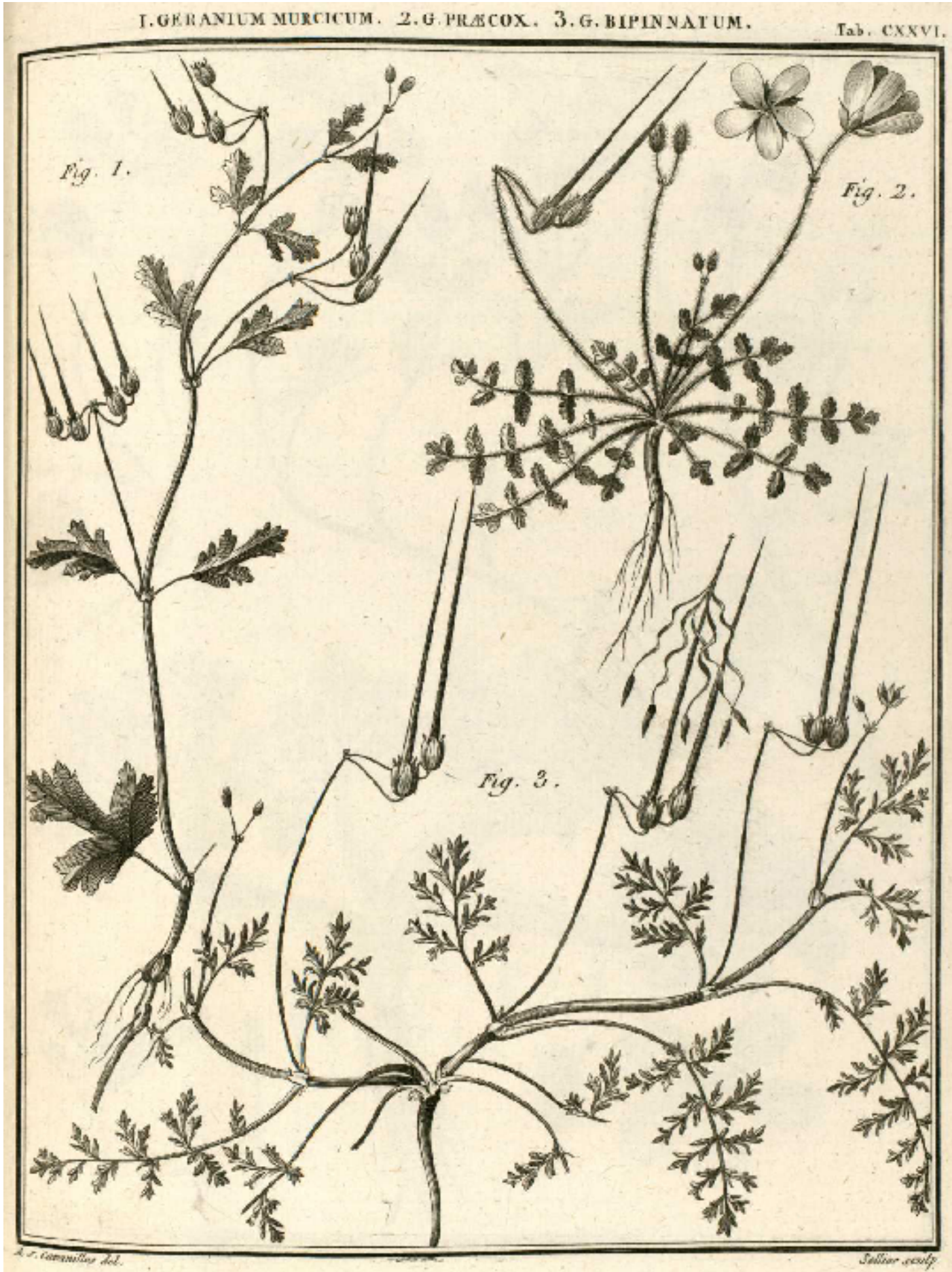

Figura 1. Lectotypus [Icon] de Geranium murcicum Cav. (Cavanilles, 1788: tab. 126, fig. 1).

Figure 1. Lectotype [Icon] of Geranium murcicum Cav. (Cavanilles, 1788: tab. 126, fig. 1). 